\title{
Ipsilateral Axillary Adenopathy from mRNA COVID-19 Vaccines
}

\author{
Shyh Poh Teo
}

Geriatrics and Palliative Unit, Department of Internal Medicine, Raja Isteri Pengiran Anak Saleha (RIPAS) Hospital, Brunei Darussalam

As older people are at risk of complications and death from severe acute respiratory syndrome coronavirus-2 (SARS-CoV-2) infections, they are an important target group for coronavirus disease 2019 (COVID-19) vaccination. ${ }^{1)}$ The mRNA vaccines BNT162b2 by Pfizer-BioNTech and mRNA-1273 by Moderna are potent COVID-19 vaccines that have demonstrated high efficacy and immunogenicity in older people. ${ }^{1,2)}$ Older people also tend to have multiple co-morbidities, which increase their risks for adverse events following immunization (AEFI) and complicate the interpretation of AEFI symptoms. Thus, post-marketing active vaccine safety surveillance is important in this population to monitor for evolving and long-term adverse events. ${ }^{3)}$

An AEFI that requires recognition by geriatricians is axillary adenopathy, particularly after the administration of mRNA vaccines. The results of a phase 3 trial showed that ipsilateral axillary adenopathy occurred in $11.6 \%$ of recipients of the first dose of the Moderna vaccine, which increased to $16.0 \%$ after the second dose in those aged 18-64 years. ${ }^{4)}$ While the Pfizer-BioNTech vaccine study did not solicit adenopathy as an adverse event, unsolicited reports of adenopathy were significantly higher in the vaccine group than in the placebo group (64 vs. 6). ${ }^{5}$ Moreover, while adenopathy was reported within 2-4 days for both mRNA vaccines, the average duration was 1-2 days for Moderna vaccine recipients compared to 10 days for Pfizer-BioNTech vaccine recipients. $\left.{ }^{46}\right)$

A systematic review of adenopathy occurring after COVID-19 vaccination reported incidence rates of $44.1 \%, 25 \%$, and $1.5 \%$ for the Pfizer-BioNTech, Moderna, and Oxford-AstraZeneca vaccines, respectively. The median elapsed times for the development of adenopathy were 12 and 5 days following the first and second doses, respectively, and persisted for up to 4 weeks post-vaccination. ${ }^{7)}$ The higher incidence of mRNA vaccine-associated adenopathy may be due to a higher immunogenic response compared to those for the other COVID-19 vaccine platforms. A case series of ipsilateral axillary swelling or adenopathy after COVID-19 vaccinations based on symptoms and physical examination found that these symptoms closely mimicked those of metastasis and lasted for up to 6 weeks. However, lymph node biopsies confirmed vaccination-related reactive lymphadenopathy. ${ }^{8)}$

Patients presenting with unilateral axillary adenopathy should be evaluated for malignancy, particularly breast cancer. Adenopathy may also be incidentally identified on routine imaging studies, such as computed tomography of the thorax or breast magnetic resonance image. Clinicians should enquire regarding recent COVID-19 vaccination, as mRNA vaccines may induce ipsilateral axillary lymph node hyperplasia. Documentation of vaccine administration, such as date and location site, should also be reviewed, as ipsilateral lymphadenopathy in the setting of recent (within 6 weeks) vaccination may indicate a benign entity. ${ }^{9}$ For patients with suspected vaccine-associated adenopathy and normal breast imaging, clinicians should consider watchful waiting, with a follow-up ultrasound to ensure resolution of lymphadenopathy performed 8 weeks after the second vaccine dose. This is a way to avoid unnecessary axillary lymph node biopsy. ${ }^{6,10)}$ However, in patients with a family history of breast cancer or a suspicion of malignancy such as breast cancer or lymphoma, lymph node biopsies should still be performed. ${ }^{11)}$

If possible, routine imaging studies should be performed before vaccination. However, in the setting of the current pandemic, COVID-19 vaccination should not be delayed. Imaging studies should be performed without delay for urgent indications such as acute symptoms or the need for urgent treatment planning, but these procedures may be may be postponed for other indications until at least 6 weeks after the second vaccine dose. Patients with primary cancer, such as breast cancer or upper limb melanoma, should ideally have both vaccine doses administered on the contralateral side. ${ }^{12)}$

In conclusion, geriatricians should be aware that ipsilateral axillary adenopathy may represent an AEFI, particularly after the administration of mRNA COVID-19 vaccines. This condition usually occurs several days after vaccination and may last for up to 6 
weeks. Expectant management may be appropriate to ensure resolution, rather than referring to further imaging studies or lymph node biopsies.

\section{ACKNOWLEDGMENTS}

\section{CONFLICT OF INTEREST}

The author claims no conflicts of interest.

\section{FUNDING}

None.

\section{REFERENCES}

1. Teo SP. Review of COVID-19 vaccines and their evidence in older adults. Ann Geriatr Med Res 2021;25:4-9.

2. Teo SP. Review of COVID-19 mRNA vaccines: BNT162b2 and mRNA-1273. J Pharm Pract 2021 Apr 12 [Epub]. https://doi. org/10.1177/08971900211009650.

3. Teo SP. COVID-19 vaccine developments and considerations for appraisal of the evidence. Pac J Med Sci 2021;21:33-5.

4. Centers for Disease Control and Prevention. Local reactions, systemic reactions, adverse events, and serious adverse events: Moderna COVID-19 vaccine [Internet]. Atlanta, GA: Centers for Disease Control and Prevention; 2020 [cited 2021 Jul 26]. Available from: https://www.cdc.gov/vaccines/covid-19/info-by-product/moderna/reactogenicity.html.

5. Centers for Disease Control and Prevention. Local reactions, systemic reactions, adverse events, and serious adverse events: Pfizer-BioNTech COVID-19 vaccine [Internet]. Atlanta, GA: Centers for Disease Control and Prevention; 2021 [cited 2021 Jul 26]. Available from: https://www.cdc.gov/vaccines/ covid-19/info-by-product/pfizer/reactogenicity.html.

6. Mehta N, Sales RM, Babagbemi K, Levy AD, McGrath AL,
Drotman M, et al. Unilateral axillary Adenopathy in the setting of COVID-19 vaccine. Clin Imaging 2021;75:12-5.

7. Keshavarz P, Yazdanpanah F, Rafiee F, Mizandari M. Lymphadenopathy following COVID-19 vaccination: imaging findings review. Acad Radiol 2021;28:1058-71.

8. Ozutemiz C, Krystosek LA, Church AL, Chauhan A, Ellermann JM, Domingo-Musibay E, et al. Lymphadenopathy in COVID-19 vaccine recipients: diagnostic dilemma in oncologic Patients. Radiology 2021;300:E296-E300.

9. Lehman CD, D'Alessandro HA, Mendoza DP, Succi MD, Kambadakone A, Lamb LR. Unilateral lymphadenopathy after COVID-19 vaccination: a practical management plan for radiologists across specialties. J Am Coll Radiol 2021;18:843-52.

10. Edmonds CE, Zuckerman SP, Conant EF. Management of Unilateral Axillary Lymphadenopathy Detected on Breast MRI in the Era of Coronavirus Disease (COVID-19) Vaccination. AJR Am J Roentgenol 2021 Feb 5 [Epub]. https://doi.org/10.2214/ AJR.21.25604.

11. Garreffa E, York J, Turnbull A, Kendrick D. Regional lymphadenopathy following COVID-19 vaccination: considerations for primary care management. Br J Gen Pract 2021;71:284-5.

12. Becker AS, Perez-Johnston R, Chikarmane SA, Chen MM, El Homsi M, Feigin KN, et al. Multidisciplinary recommendations regarding post-vaccine adenopathy and radiologic imaging: Radiology Scientific Expert Panel. Radiology 2021;300:E323-E327.

Corresponding Author: Shyh Poh Teo, FRACP

Geriatrics and Palliative Unit, Department of Internal Medicine, Raja Isteri Pengiran Anak Saleha (RIPAS) Hospital, Jalan Putera Al-Muhtadee Billah, Bandar Seri Begawan, BA1710, Brunei Darussalam

E-mail: shyhpoh.teo@moh.gov.bn

ORCID: https://orcid.org/0000-0002-6117-5774

Received:June 10, 2021; Revised: July 19, 2021; Accepted:July 21, 2021 